\title{
Status of the DNB based ITER CXRS and BES diagnostic
}

\author{
M. von Hellermann and R. Jaspers \\ FOM-Institute for Plasma Physics "Rijnhuizen," Association EURATOM, Trilateral Euregio Cluster, 3430BE \\ Nieuwegein, Netherlands
}

W. Biel, A. Litnovsky, O. Neubauer, and M. Pap

Institut für Plasmaphysik, Forschungszentrum Jülich GmbH, EURATOM Association, Trilateral Euregio Cluster, D-52425 Jülich, Germany

N. C. Hawkes, C. Marren, and B. Walton

UKAEA Culham Laboratory EURATOM Association, Abingdon, OX14 3CB United Kingdom

Y. Kaschuck, V. Serov, and S. Tugarinov

TRINITI Troitsk, 14190 Troitsk, Moscow Region, Russia

W. Vliegenthart and K. Moddemeijer

TNO Science and Industry, Stieltjesweg 1, 2600 AD, Delft, Netherlands

C. Walker and C. Ingesson

ITER IT Garching, P.O. Box 1322, 85741 Garchimg, Federal Republic of Germany

(Received 8 May 2006; presented on 11 May 2006; accepted 16 June 2006; published online 29 September 2006)

A status report is given on recent joint activities on the ITER CXRS and BES diagnostic package. Expected measurement performances are reviewed as well as comprehensive discussions are led on an integral approach to the implementation of Core and Edge CXRS observation periscopes. The "first mirror" location, its operational temperature, maintenance issues, and optimization of optical imaging are addressed. In parallel to more technical aspects, particular attention has also been given to the development of common evaluation and modeling tools. One part of this work is linked to the modeling of spectra for existing fusion devices and their CXRS diagnostics and extrapolation to the ITER environment. The purpose of this effort is to provide tools for the optimization of spectroscopic instrumentation, and moreover, the specifications of a suitable diagnostic beam. (C) 2006 American Institute of Physics. [DOI: 10.1063/1.2229222]

\section{INTRODUCTION}

The conceptual reports on the ITER CXRS and BES diagnostic ${ }^{1-3}$ have summarized its main features and measuring capabilities. More recent work reviews the overall concept and performance studies, emphasizes the aspects of the integrated design of the CXRS diagnostic in ITER, and addresses critical issues in the preparation of the actual construction phase. A further goal is a review of the entire system and its measuring capabilities in view of recent developments. Since the responsibility for the ITER CXRS is shared presently between the RF (equatorial port periscopes viewing the outer half of the plasma profile) and the EU (upper port periscope viewing the plasma core region) a close collaboration has evolved including common workshops. ${ }^{4}$ Within the EU the work carried out for this review is based on intense discussions and exchange of ideas between the three involved associations UKAEA, FZJ, and FOM. In view of the preparatory character of the present phase it was essential to ensure a consistency of the various subtasks such as the "first mirror," shutter, optical layout, fiber link, and instrumentation. Several essential features of a common strategy evolved.

${ }^{\text {a) URL: www.fom.rijnh.nl }}$

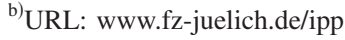

(a) Minimization of radiation and neutron load on first mirror, hence the recommendation of a retracted position towards the rear of the blanket structure rather than close to its front side.

(b) Preservation of oblique observation geometry required for Doppler measurements of DNB Balmer-Alpha emission, needed for verification of major radii allocated to each radial channel.

(c) Identification of upper port 2 as alternative solution to the reference port 3 promising to satisfy conditions (a) and (b) and also leading to increased sensitivity for toroidal rotation and magnetic pitch angle measurements.

(d) Implementation of a shutter in front of the first mirror, in order to minimize first mirror contamination during beam-off phases, e.g., during cleaning discharges.

(e) The mounting of mirrors on heat-exchange support structure, at a temperature level above environment temperature $\left(450{ }^{\circ} \mathrm{C}\right)$, in order to minimize deposition of contaminants

(f) Feasibility study of a retractable of mirror tube concept, in particular, for first mirror and shutter, to provide the means for easy maintenance.

(g) Alignment schemes following prealignment in the assembly phase to be based on fine adjustments by rota- 


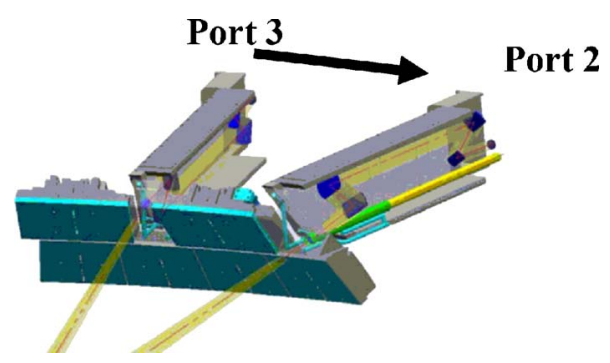

FIG. 1. Retraction of first mirror towards rear of blanket structure requires a port swap from port 3 to port 2 in order to retain a Doppler shift of the Balmer-Alpha beam spectrum [i.e., the angle between line of sight and DNB should not be $90^{\circ}$. The two dog-leg optical labyrinth is indicated schematically; first mirror and shutter share an extractable pipe.

tion of mirror, BES signal optimization using fine adjustments of fiber bundle position at the end of the periscope, and finally, the use of in vessel inspection cameras. The latter scheme requires high quality inverse imaging optical properties of the observation periscope. A TV inspection will identify target locations on the opposing wall of individual fibers making use of laser light fed in from the spectrometer side.

(h) A desirable potential extension of the reference core observation region $r / a<0.5$ to the entire region ( 0 $<r / a<1.0$ ). The rationale behind this extension is to achieve the largest possible overlap between the upperport Core-CXRS periscope and the equatorial-port Edge-CXRS periscopes. The physics reasons are, firstly, the provision of fiducial cross-checks for poloidal velocity measurements, a possible correction for the gyro-orbit effect, ${ }^{5}$ and, secondly, the investigation of anisotropic features, e.g., alpha particle slowingdown features.

\section{A. Periscope issues}

The first mirror of the CX observation periscope is exposed to a substantial radiation level. Present estimates indicate values of the order $2 \mathrm{~W} / \mathrm{cm}^{3}$ for $13.5 \mathrm{MeV}$ neutrons corresponding to a flux of $7 \times 10^{12} n \mathrm{~s}^{-1} \mathrm{~cm}^{-2}$ close to the blanket surface. At this power load a temperature increase of the order $1{ }^{\circ} \mathrm{C} / \mathrm{s}$ would lead during a $1000 \mathrm{~s}$ ITER pulse to a substantial warming up of the mirror. This implies either active cooling of the mirror, or alternatively, keeping the mirror at a controlled temperature level (link to heat exchange system). At the same time, and this is a further concern, the mirror temperature should be higher than its immediate vicinity, in order to minimize particle deposition. The recommended temperature level is $450{ }^{\circ} \mathrm{C}$, and as the most promising mirror material presently either monocrystalline molybdenum or rhodium mirrors are under discussion. ${ }^{6}$

If the first mirror could be retracted towards the inside of the blanket structure, this would reduce substantially the neutron load and would also lead potentially, due to the viewing slot cut into the blanket structure, to a reduction of the particle flux. Unfortunately, a first mirror position in a retracted location behind the blanket in upper port 3 is accompanied by viewing angles incompatible with the physics requirement for a measurable Doppler shift of the DNB emission spec-

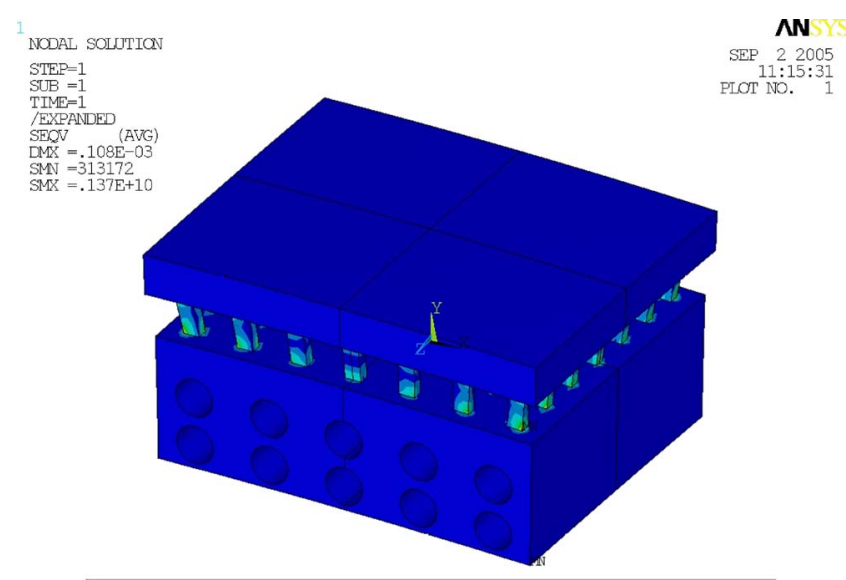

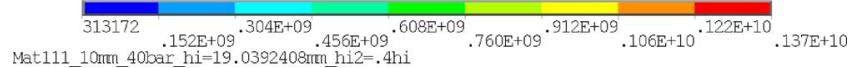

FIG. 2. Principle design of the first mirror system with the mirror surface, the intermediate layer, and the cooling block with water channels. The cooling block is at $100{ }^{\circ} \mathrm{C}$, and additional electric heating is added to the actual mirror keeping at about $400{ }^{\circ} \mathrm{C}$. Stress distribution $\left(\mathrm{N} / \mathrm{m}^{2}\right)$ is shown for maximum heating from plasma radiation and neutrons.

trum (cf. Ref. 2, Figs. 10 and 11). This requirement implies nonperpendicular views of the DNB path as seen from the CXRS periscope. This consideration led subsequently to the proposal to shift the CXRS diagnostic from the foreseen upper port 3 towards the neighboring upper port 2 (see Fig. 1). For this port, a first mirror in a retracted location behind the blanket will sustain the measuring capability and ensure at the same time an enhanced lifetime of the first mirror due to its more protected position. Since a cut of an observation channel through the adjacent blanket structures of the CXRS periscope is technically prohibitive, the maximum retraction depth is limited to a maximum of about $300 \mathrm{~mm}$.

The basic design idea of the first mirror consists of the mirror surface, the cooling body with cooling holes bored into it, and an interlayer between both parts. Preferably cooling is provided by the available water cooling circuit, whereas heating is provided by electrical current. Heat resistance between mirror and cooling panel has to maintain temperature gradient $\left(400-100{ }^{\circ} \mathrm{C}\right)$ at maximum heating from plasma and neutrons.

A parameter study has been done for different geometries and materials. Heat transfer and bending at maximum plasma heating have been analyzed. For the calculations, made with the FEM code ANSYS, we determined the cooling block to have the schematic design as described in Fig. 2. The result of the stress studies is a maximum bending in the center of the flat mirror of the order of $10^{-6}$ and will therefore not affect the imaging properties noticeably.

The physics demands for common fiducial points of the upper port and equatorial port viewing section, that is the need for an extended DNB view, on the one hand, and also the recognition that imaging properties of the periscope need to be reviewed in terms of spatial resolution and inverse imaging have led to a substantial change of the original layout. The new proposal for the upper port periscope envisages a system which has only one mirror "dog leg" and is followed by a Cassegrain output in front of the fiber bundle (Figs. 3 and 4). A ray-tracing (Zemax) test of the inverse 


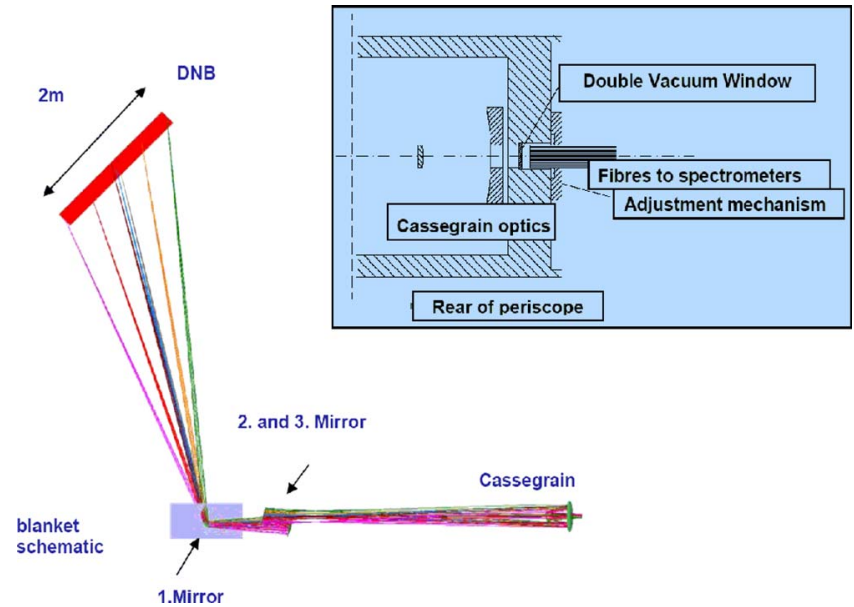

FIG. 3. Alternative Cassegrain optics for upper port viewer enabling improved imaging of entire beam section $(0<r / a<1)$. Inlet: fiber bundle at focal point of Cassegrain with fine adjustment capability for alignment on DNB (schematic).

imaging properties shows that due the Cassegrain optics a $1 \mathrm{~mm}$ fiber which is imaged into the plasma will have a spot size of approximately $25 \mathrm{~mm}$. This corresponds experimentally to the scenario where a helium laser is shone from the spectrometer side through a single fiber, to be used as an alignment check for the periscope. Another result of the raytracing study is the alignment capability which is tolerated by the periscope assembly in the case fine-tuning the position of the receiving fiber bundle with respect to the Cassegrain output (cf. Fig. 4 inlet).

A similar review of the equatorial periscope (Fig. 5) envisages a combination of mirrors and correction lenses ensuring optimized imaging properties. Due to the different viewing angles and geometric intersection of magnetic flux surfaces the equatorial view an almost tangential intersection and therefore maximum radial resolution, and in contrast for the upper port view resolution is best in the very plasma core (Fig. 6).

\section{B. Spectral modeling}

Spectral modeling has developed into an indispensable tool for quantitative spectroscopy and the analysis of complex CX spectra, cf. Ref. 8 With respect to a review of measurement capabilities of the ITER CXRS diagnostic the main challenge in modeling is the assessment of the spectral

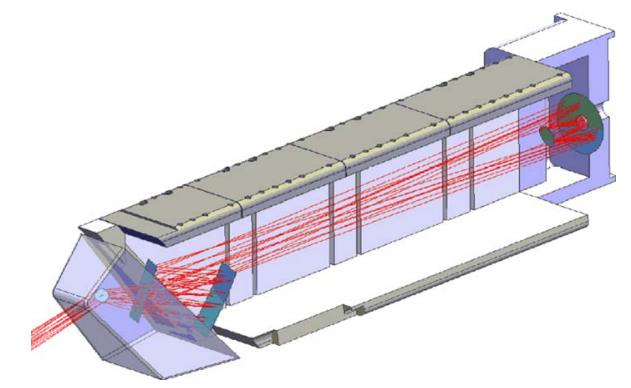

FIG. 4. Schematic layout of Cassegrain optics periscope in upper port 2 with first mirror in slightly retracted position $(200 \mathrm{~mm})$ avoiding thus cutting through adjacent blanket structures.

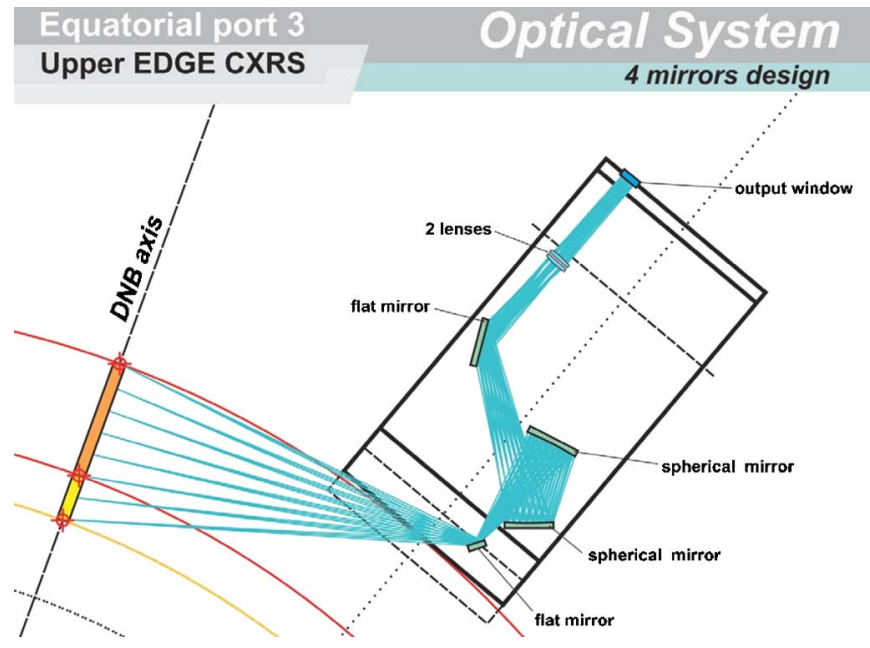

FIG. 5. Advanced equatorial port periscope design with extended view of DNB covering $(0.3<r / a<1)$. The lower limit is a minimum threshold for signal detection for He II (helium ash measurement) with SNR $>10$. A combined mirror and lenses assembly improves imaging properties and enables high spatial resolution required for barrier studies $(\delta r<a / 40)$, see also Fig. 6.

signal-to-noise ratio. The creation of synthetic CX spectra encompasses automatically the need for a quantitative description of additional background features, which consist one the one hand on a huge level of continuum radiation and two types of passive background line emission: narrow, low temperature lines representing the very plasma edge, and the second passive charge exchange line emission representing the interaction of neutral hydrogen recycling from the wall and fully stripped impurity ions. ${ }^{7}$ In both cases it is difficult to achieve a tolerable level of accuracy for absolute intensities, since in each case it is difficult to model reliably the donor density close to the plasma boundary. In contrast, precise wavelength positions of edge lines are well documented, and temperatures are not too critical.

In theory, any passive background feature can be sup-

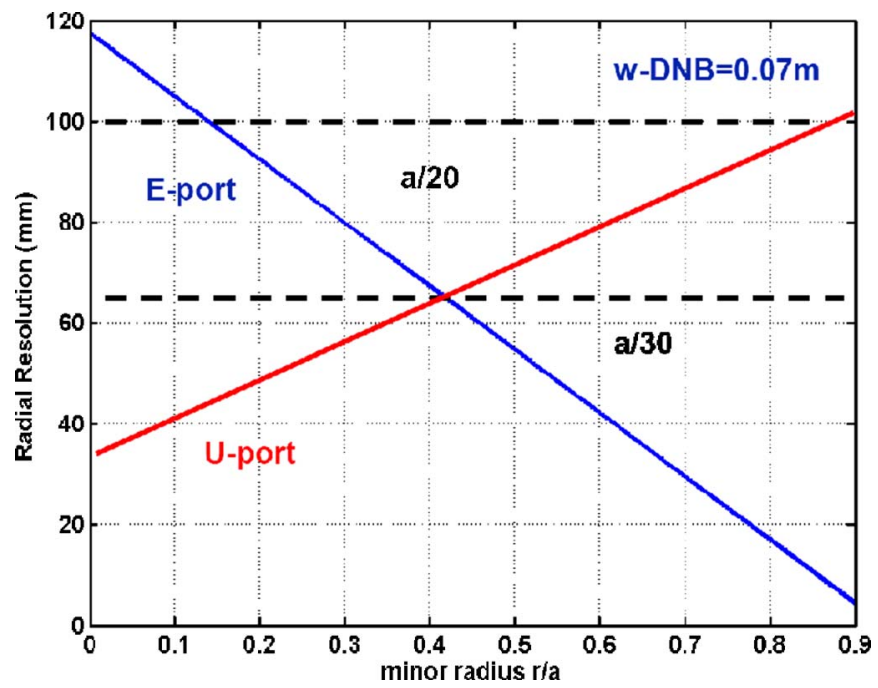

FIG. 6. Spatial resolution for both upper port and equatorial port systems based on geometric slab model of DNB assuming a $1 / e$ radius of $0.07 \mathrm{~m}$ corresponding to a DNB divergence of $4.5 \mathrm{mrad}$. The ITER measurement requirement table specifies $a / 30$ resolution. 


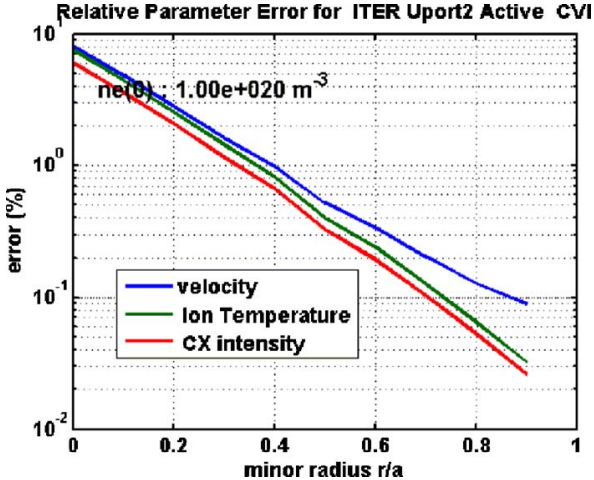

FIG. 7. Upper port error analysis based on simulated CVI CX spectra where main noise factor is given by underlying continuum radiation. The ITER reference scenario is $n_{e}(0)=10^{20} \mathrm{~m}^{-3}, T_{e}=T_{i}=15 \mathrm{keV}$, and $n_{C} / n_{e}=0.01$, assuming flattop density profiles and parabolic temperature profiles. It is assumed that passive background line emission such as lines emitted close to the plasma wall and passive CX emission may be subtracted by either applying DNB modulation or providing suitable passive viewing lines. Alternatively, a fully modeled synthetic PCX spectrum may be added which does not change significantly single-Gaussian errors (cf. Ref. 7).

pressed by beam modulation. This recipe fails, however, in the case, when during the beam-on phase the neutral density background is changed compared to the beam-off phase. An alternative, or complementary, scheme is to implement an additional set of "passive" lines of sight which are directed to the immediate background close to the beam area, thus monitoring wall areas close to the active line of sight set, but avoiding direct light collection from the beam itself.

In the case of the JET CXRS diagnostic (see Refs. 5 and 6) the line shape of the passive CX spectrum is successfully approximated by a Gaussian spectral shape with a temperature and rotation corresponding to a minor radius of $r / a$ $=0.95$. Moreover, switching of the JET (heating) neutral beams does affect the ionization balance and hence the boundary neutral density. As a result, beam modulation does not give the same results as the evaluation of a comprehensive spectrum containing active and passive components without beam modulation.

In the following (Fig. 7) we present the result of a simulation study where the CX signal and the continuum background are calculated for the ITER reference scenario and a single active CX component is extracted by a least-square minimization, assuming photon noise as the dominant noise feature. The ITER measurement requirements are clearly met, even in the very core region $(r / a<0.3)$, where the spectral signal-to-noise ratio (SNR) for He II drops to estimated levels around 5; however, the least-square minimization errors which are determined by the entire spectrum are of the order $10 \%$ even on axis. In the case of nonadequate beam modulation where the passive $\mathrm{CX}$ features may not be completely suppressed, the expected errors for central He II intensities are still in an acceptable range, and therefore the main goal of the ITER CXRS diagnostic of providing core helium ion densities will be achieved. On the modeling side, more work on the prediction of local neutral densities, which is a nonisotropic feature, will help to move to more precise predictions of the backgound emission levels.

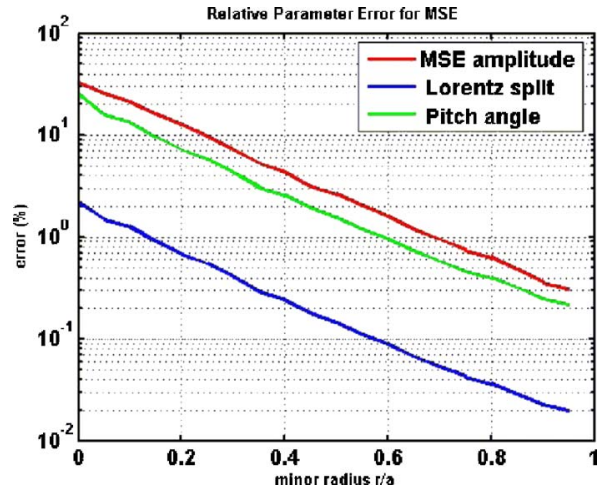

FIG. 8. Upper port error analysis for MSE and BES signal evaluation based on the same reference scenario, the magnetic field is $5.2 \mathrm{~T}$, and a parabolic $q$ profile has been assumed.

The parametric simulation model allows the investigation of critical parameter dependencies encompassing beam parameters (beam energy, neutral current, species mix, and beam divergence), viewing geometry (solid angles, line of sight directions, and path length through plasma), spectral instrumentation (optical throughput, spectral resolution, quantum efficiency, and integration time), plasma data $\left(n_{e}\right.$, $n_{i}, n_{z}, T_{e}, T_{i}, v_{\text {rot }}$, and $B_{t}$ ), and modeled edge features (edge temperatures).

A similar exercise as for the thermal CX feature representing the dominant low- $Z$ impurity ions has been performed for beam emission spectroscopy, with regard to local beam densities, pitch angles, and Lorentz splitting as a measure of the total local field (Fig. 8). The potential measurement of local fuel mixtures is investigated by a simulation of thermal CX spectra for deuterons and tritons.

A result of the combined CXRS and BES simulation work, it has become clear that task specific spectral instruments will be needed. For example, a moderate spectral resolution of about $0.2 \mathrm{~nm}$ for the measurement of broadband thermal CX features, and for the evaluation of the Stark multiplet with its ensemble of nine well separated narrow features a significantly higher resolution of about $0.02 \mathrm{~nm}$ will be needed.

${ }^{1}$ M. G. von Hellermann, W. G. F. Core, A. Howman, C. Jupen, R. W. T. König, M. F. Stamp, H. P. Summers, P. R. Thomas, and K.-D. Zastrow, First International Workshop on ITER Diagnostics, Varenna, September 1995 in Diagnostics for Experimental Fusion Reactors I (Plenum, New York, 1996), edited by P. E. Stott, A. Wootton, G. Gorini, and E. Sindoni, pp. 321.

${ }^{2}$ M. von Hellermann (EFDA Contract No. 01.649; not publicly available, contact:mgvh@rijnh.nl).

${ }^{3}$ A. Malaquias et al., Rev. Sci. Instrum. 75, 3393 (2004).

${ }^{4}$ Joint Workshop on ITER CXRS at TRINITI, Troitsk, RF, October 2005 (unpublished); proceedings see tugar@triniti.ru.

${ }^{5}$ R. E. Bell, M. C. Zarnstorff, and E. J. Synakowski, PPPL 3447 (2000).

${ }^{6}$ A. Litnovsky et al., Proceedings of the 32nd EPS Conference on Plasma Physics, Tarragona, Spain, 27 June - 1 July 2005, ECA, Vol. 29C, P-1.015 (2005).

${ }^{7}$ M. Tunklev, P. Breger, K. Günther, M. von Hellermann, R. König, M. O'Mullane, and K.-D. Zastrow, Plasma Phys. Controlled Fusion 41, 985 (1999).

${ }^{8}$ M. G. von Hellermann, W. Biel, G. Bertschinger, C. Jupen, M. O'Mullane, H. Summers, and A. Whiteford, Phys. Scr., T 120, 19-29 (2005) 